\title{
Renal cell carcinoma with nephrotic syndrome: a case report and literature review
}

\author{
Fei Song, Chuan Liu, Junyong Zhang, Zili Hu \\ Department of Urology, The Second Affiliated Hospital of Chongqing Medical University, Chongqing 400010, China \\ Correspondence to: Chuan Liu. Department of Urology, The Second Affiliated Hospital of Chongqing Medical University, Chongqing 400010, China. \\ Email: liuchuan100@hospital.cqmu.edu.cn.
}

\begin{abstract}
In previous studies, the association between malignant tumors and the development of glomerular disease has been well documented. Malignant tumors that cause glomerular damage are mainly classified into two categories: Hematological tumors and solid tumors. Of them, the most common type is Hodgkin's lymphoma, while renal carcinoma is substantially infrequent. This study describes a 67-yearold Chinese male patient who was hospitalized for nephrotic syndrome with the symptoms of hypertension, edema and foamy urine, and has not taken any related drugs before. In the process of hospitalization, a renal carcinoma in the stage T1N0M0 was suspected by abdominal enhanced computed tomography scan. What is unique about this patient is the treatment-removing the tumor first may aggravate the kidney damage, which leads to further deterioration of renal function and using hormone drugs to treat nephrotic syndrome may cause tumor enlargement. Ultimately, the patient underwent the surgery of laparoscopic partial nephrectomy and took pathological examinations of the renal neoplasm and the normal tissue next to the tumor. Based on the pathological results, renal cell carcinoma with membranous nephropathy (MN) was verified. After reviewing the case reports of renal cancer with glomerular disease in the past 50 years, it is hoped to provide a basis for the standardized diagnosis and treatment of this combination disease in renal.
\end{abstract}

Keywords: Renal cell carcinoma; paraneoplastic glomerular disease; nephrotic syndrome; membranous nephropathy $(\mathrm{MN})$; IgA nephropathy; case report

Submitted Nov 04, 2019. Accepted for publication Mar 06, 2020.

doi: 10.21037/tau.2020.03.41

View this article at: http://dx.doi.org/10.21037/tau.2020.03.41

\section{Introduction}

In 1922, Galloway introduced the concept of paraneoplastic glomerular disease (1). Paraneoplastic glomerulopathy refers to glomerular lesions that are not directly related to tumor burden, invasion or metastasis, but are induced by tumor cell products such as hormones, growth factors, cytokines and tumor antigens (2). In 1966, the association between malignant tumors and glomerular disease was initially identified (3). The malignant tumors causing this damage are mainly divided into two types: Hematological malignancies incorporating Hodgkin's lymphoma usually associated with micro-kidney disease, and solid tumors mainly including lung cancers and digestive tract tumors which usually lead to $\mathrm{MN}(3,4)$. However, reports of this glomerular damage caused by renal cancer are rare.

The treatment of paraneoplastic glomerulonephritis is greatly different from the treatment of idiopathic glomerulonephritis. Therefore, it is clinically crucial to recognize paraneoplastic glomerulonephritis. In general, if the glomerular disease derived from a malignant tumor, the symptoms of glomerular disease may be relieving with the resection of the tumor lesion, and the diagnosis of paraneoplastic glomerulonephritis should be considered. Here we report a case of glomerular disease caused by renal cancer. What is unique about this case is the treatmentno matter which disease is treated first, there is a big risk. And at the same time, we collected relevant case reports for nearly 50 years, hoping to provide a basis for the standardized diagnosis and treatment of renal cell 


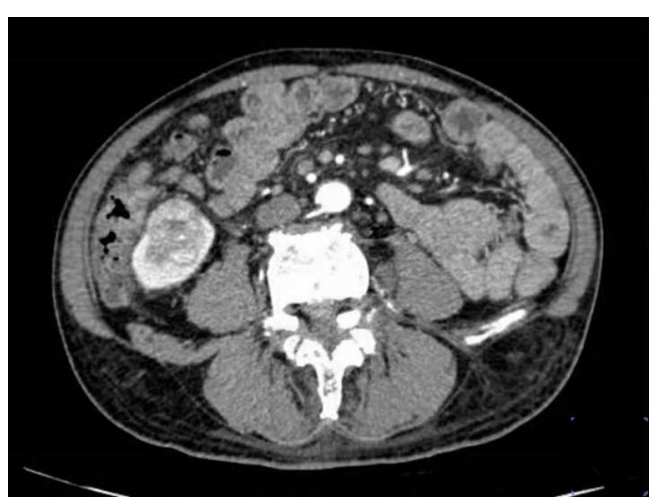

Figure 1 Abdominal enhanced CT shows uneven enhancement of the right renal inferior mass artery.

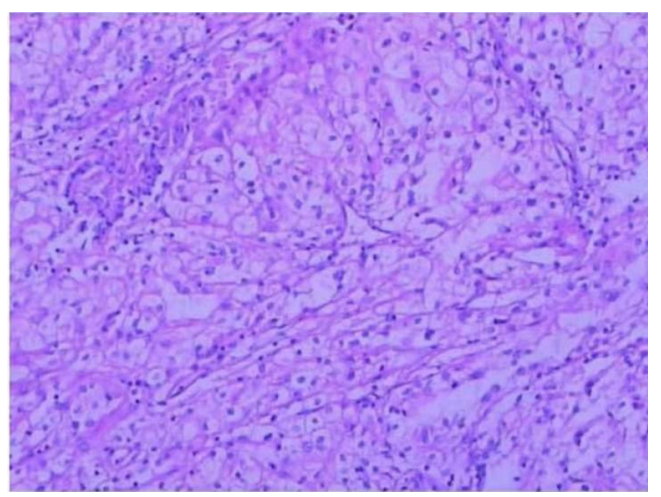

Figure 2 Renal tumor tissue under light microscopy can be seen mainly composed of clear cells (HE staining, $\times 400$ ).

carcinoma with glomerular disease and exploring the potential pathophysiological mechanism of paraneoplastic glomerulonephritis.

Nephrotic syndrome is a form of hypoproteinemia and edema caused by massive loss of urine protein, which is one of the most common clinical manifestations of glomerular disease (5). Renal cancer is one of the 10 most common cancers, accounting for $3.7 \%$ of new-onset tumors and the third among urinary malignancies. Because there is no specific clinical symptom in the early stage of the disease, early diagnosis is highly difficult, while it is mostly found accidentally during routine physical examinations (6). Here, we report a case of a renal cancer patient who was hospitalized with symptoms of nephrotic syndrome. The case study was approved by the Ethics Committee of The Second Affiliated Hospital of Chongqing Medical University, and the patient provided informed consent.

\section{Case presentation}

\section{History}

A 69-year-old Chinese male was hospitalized due to the symptom of edema for 3 months in November 2018. The patient developed edema of the eyelids and lower extremities 3 months ago, with foamed urination, gross hematuria, 20 years' previous history of hypertension and no back pain. Physical examination: blood pressure $155 / 91 \mathrm{mmHg}(1 \mathrm{mmHg}=0.133 \mathrm{kPa})$, moderate depression edema in both lower extremities. Laboratory examination: albumin $22 \mathrm{~g} / \mathrm{L}$, total cholesterol $7.25 \mathrm{mmol} / \mathrm{L}$, triglyceride $1.85 \mathrm{mmol} / \mathrm{L}$, erythrocyte sedimentation rate $50 \mathrm{~mm} / \mathrm{h}$, creatinine $66 \mu \mathrm{mol} / \mathrm{L}$, glomerular filtration rate: left $37.5 \mathrm{~mL} / \mathrm{min}$, right $33.8 \mathrm{~mL} / \mathrm{min}$, antiphospholipid syndrome antibody spectrum, rheumatoid factor, antiGlomerular basement membrane antibody, autoantibody, anti-nuclear antibody, vasculitis antibody spectrum were negative, immunoglobulin, complement 3, complement 4 were normal. The urinary red blood cell count was $35.9 / \mu \mathrm{L}$, and the protein type was found in the urine sediment analysis. The total urine protein level was $24.8 \mathrm{~g} / 24 \mathrm{~h}$. An abdominal enhanced computed tomography scan showed a $3.2 \mathrm{~cm} \times 2.7 \mathrm{~cm}$ mass in the right lower kidney and uneven enhancement in the arterial phase (Figure 1). He was diagnosed clinically with nephrotic syndrome and right kidney tumor, taking no any possible pathogenic drugs before.

Because the etiology of nephrotic syndrome is unknown, it cannot be clear whether the renal tumor induced the glomerular disease or not; further more, hormone therapy for nephrotic syndrome is a contraindication for cancer patients, it can lead to further tumor growth. Therefore, it is impossible to treat nephrotic syndrome first. At the same time, the patient's glomerular filtration rate is low, which signifies if radical nephrectomy is performed, renal insufficiency may occur after surgery great possibility. Thus, after the discussion of the department, laparoscopic partial nephrectomy is performed. Postoperative pathological examination: the size of the right renal mass was $3.0 \mathrm{~cm}$ $\times 2.8 \mathrm{~cm} \times 2.5 \mathrm{~cm}$. Microscopically, the tumor cells were observed to be transparent, conforming to renal clear cell carcinoma, and locally invading the capsule, which did not break through (Figure 2). Immunohistochemical staining: CK8 (++), PAX-2 (+), EMA (++), Vim (+), CD10 (++), CK7 individual cells (+), CDK20 (-), E-Cal (+), CD117 (-), CEA (-), P504S (++). The postoperative pathological stage 
was T1aN0M0. To determine the etiology of nephrotic syndrome, a small amount of normal renal tissue specimens were taken for pathological examination: under light microscope: Glomerular focal segmental mild mesangial hyperplasia (4/40), diffuse thickening of the basement membrane $(35 / 40)$, spheroidal sclerosis $(5 / 40)$, tubular

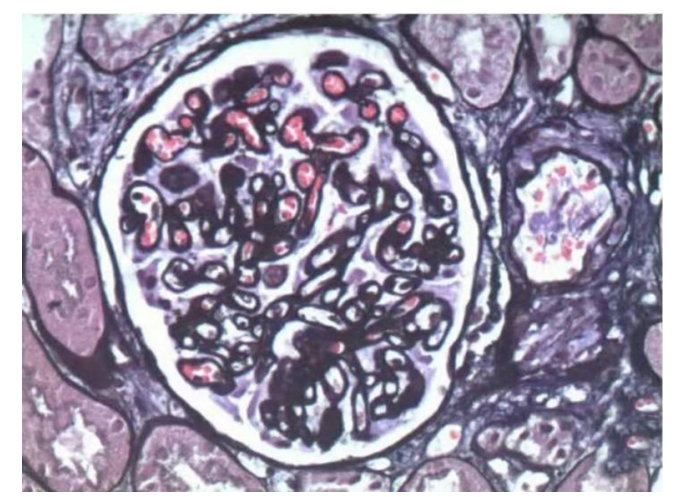

Figure 3 Renal glomerular basement membrane diffuse thickening under light microscope (PASM staining, ×400).

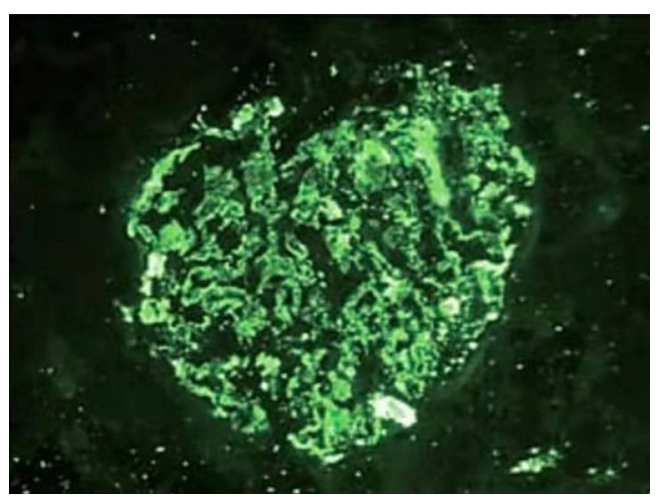

Figure 4 Immunofluorescence staining suggests fine particle-like deposition of $\mathrm{IgG}$ along the glomerular basement membrane (antiIgG staining, ×400). epithelial cell turbidity and granular degeneration, protein tube type inside (Figure 3); immunofluorescence: IgA: (++); IgG: (+); IgM(+); C3: (+); Fibrin(++); C1q: (++), immune complex deposition were found in mesangial and capillary vasospasm (Figure 4). Diagnosed as MN.

During 1 year of follow-up, the patient accepted monthly review of relevant indicators, and did not get any medication for nephrotic syndrome. It is presented that the indicators still gradually improved. The lower extremity edema disappeared, the serum albumin rose to $31.6 \mathrm{~g} / \mathrm{L}$, the 24-hour urine protein decreased to $3.8 \mathrm{~g} / 24 \mathrm{~h}$, and the glomerular filtration rate was $47.8 \mathrm{~mL} / \mathrm{min}$ in the left and $32.1 \mathrm{~mL} / \mathrm{min}$ in the right. There were no major complications noted, no tumor lesions remained and no signs of relapse found (Table 1). We believe that the patient's glomerular damage has been completely improved with the resection of the primary tumor.

The patient is extremely satisfied with the treatment on account of not only the minimally invasive operation as well as fast recovery, but also the preserved kidney function. And after surgery, the physical condition has been greatly improved.

\section{Discussion}

Most cancer patients may have glomerular diseases (7). The combination of cancer and $\mathrm{MN}$ is the most common, and a meta-analysis has estimated that the prevalence of cancer in $\mathrm{MN}$ patients is estimated to be $10 \%$ (8). However, it is difficult to assess the true prevalence of cancer-related glomerular disease, mainly for the following reasons: (I) potential detection bias (e.g., patients with MN may be more aggressive in screening for cancer); (II) population characteristics of the population (e.g., MN and cancers occur more frequently in older people and/or smokers); (III) most drugs used to treat glomerular diseases are potential carcinogenic drugs, therefore possibly leading to subsequent

Table 1 The timeline of this patient

\begin{tabular}{|c|c|c|c|c|c|}
\hline Index & Before surgery & $\begin{array}{l}\text { Postoperatively } \\
3 \text { months }\end{array}$ & $\begin{array}{c}\text { Postoperatively } \\
6 \text { months }\end{array}$ & $\begin{array}{l}\text { Postoperatively } \\
9 \text { months }\end{array}$ & $\begin{array}{l}\text { Postoperatively } \\
12 \text { months }\end{array}$ \\
\hline Edma & Severe & None & None & None & None \\
\hline Urine protein, g/24 h & 24.8 & 14.3 & 9.4 & 6.6 & 3.8 \\
\hline
\end{tabular}


Table 2 Literature review of the 12 cases of renal cell carcinoma with nephrotic syndrome to date

\begin{tabular}{lcccccc}
\hline No. (ref.) & Sex & Age & Tumor size $(\mathrm{cm})$ & Surgery & Nephrosis & Follow-up \\
\hline $1(9)$ & M & 63 & Not described & Not described & MN & Not described \\
$2(10)$ & M & 62 & $7.0 \times 6.5$ & Radical nephrectomy & MN & Nephrosis recovery \\
$3(11)$ & F & 77 & 12 & Radical nephrectomy & MN & Nephrosis recovery \\
$4(12)$ & M & 58 & 5 & Radical nephrectomy & MN & Not described \\
$5(13)$ & F & 76 & 4 & Radical nephrectomy & MN & Death due to heart disease \\
$6(14)$ & M & 62 & 1.6 & Partial nephrectomy & MN & Nephrosis recovery \\
$7(15)$ & M & 55 & Not described & Radical nephrectomy & MN & Nephrosis recovery \\
$8(16)$ & M & 58 & $7.5 \times 6$ & Not described & IgA & Nephrosis recovery \\
$9(16)$ & M & 66 & $3 \times 2.6$ & Not described & IgA & Dialysis after 1 year \\
$10(16)$ & M & 59 & $2.1 \times 1.1$ & Not described & IgA & Nephrosis recovery \\
$11(17)$ & F & 29 & $2 \times 2$ & Partial nephrectomy & MCD & Nephrosis recovery \\
$12(18)$ & F & 64 & $7 \times 7$ & Radical nephrectomy & MCD & Not described \\
\hline
\end{tabular}

MN, membranous nephropathy.

malignancies. Therefore, it is no surprise that there is little information about the prevalence of paraneoplastic glomerular.

In a review of previous studies conducted over the past 50 years, a total of 10 reports were collected and 12 patients with renal cancer plus nephrotic syndrome were: $7 \mathrm{MN}$ (9-15), 3 IgA Kidney disease (16), 2 cases of tiny kidney disease $(17,18)$ (Table 2).

Although the number of confirmed cases is limited, clinical features such as close temporal relationships and parallel evolution (improvement, regression, recurrence) can still confirm the association between solid tumors and kidney diseases. It has been widely maintained that hematological malignancies cause micro-kidney diseases, and solid tumors usually lead to $\mathrm{MN}(3,4)$. This study demonstrates that glomerular diseases of the same pathological type can be found in different types of tumors, and glomerular diseases of different pathological types can also occur in the same type of tumors.

The pathogenesis of tumor-associated nephropathy is still undetermined, and changes in immune responses appear to play a role in the pathogenesis. This view is supported by studies in Buffalo/Mna rats in which TH2 polarization induces thymoma-associated minimal change disease (MCD) and focal segmental glomerular sclerosis (FSGS) (19), and there are also animal models showing that in tumor-bearing animals, large amounts of IgG deposits and loss of glomerular foot processes may lead to proteinuria and glomerular damage (20).

However, through clinical research, a certain number of possible mechanisms have been drawn.

MN: manifested as diffuse deposits under the epithelium leading to uniform thickening of the glomerular capillary wall.

(I) It is believed that the relevant antigen is released from the surface of the tumor cell, thereby stimulating the production of the antibody. Subsequently, an antigen-antibody immune complex can be formed deposited in the glomerulus. Some reports have confirmed the presence of tumor antigens and/or specific antibodies in the glomeruli of patients with paraneoplastic glomerular disease. The tumor antigens described include cancer embryo antigen (21), prostate specific antigen (22), melanoma antigen (23), and unrecognized tumor antigen (24), which provides evidences for the role of immune complexes in paraneoplastic glomerulopathy;

(II) malignant tumors change the immune status of the body, making the body more susceptible to endogenous antigens and triggering antigenantibody reactions and in situ binding (25).

IgA nephropathy: diffuse IgA deposition in the mesentery. 
It is speculated that malignant tumor invasion of the mucosa leads to an increase in circulating IgA levels, thus forming mesangial deposits (26).

MCD: no lesions were formed under light microscope, and glomerular epithelial cells disappeared under electron microscope.

This may be caused by cytokines secreted by infiltrating lymphocytes and macrophages, which increases the transmission of glomeruli. It has been reported that a patient with rectal adenocarcinoma plus micronephropathy has elevated levels of vascular endothelial growth factor (VEGF). After tumor resection, proteinuria disappeared and VEGF levels fell to normal (27).

$\mathrm{MN}$ is most closely related to solid malignant tumors in glomerular diseases, with an incidence of about $10 \%$ (8). Lung cancer, gastric cancer, and prostate cancer are the most commonly reported tumor types, while renal cancer is rare. And it is difficult to verify that $\mathrm{MN}$ is primary or secondary in clinic; nevertheless, this is highly necessary, because blindly applied drugs for the treatment of nephrotic syndrome may lead to tumor enlargement (16). Although there are no specific signs or symptoms, some clues may suggest tumor-associated $\mathrm{MN}$ : (I) abnormality in tumor marker screening (II) deposition of IgG 1 and IgG 2 subtypes in renal tissue; (III) renal tissue There are more than 8 inflammatory cells in the glomerulus: studies have shown that the diagnostic threshold of tumorassociated $\mathrm{MN}$ and primary $\mathrm{MN}$ is distinguished by the infiltration of 8 inflammatory cells in the glomerulus as a cut-off value, with a specificity of $75 \%$ with a sensitivity of $92 \%$; (IV) anti-PLA2R antibody negative in blood or kidney tissue: podocyte transmembrane glycoprotein $M$ type antiphospholipid 2 receptor (anti-PLA2R antibody) autoantibody. Seventy percent to eighty-two percent of patients with primary $\mathrm{MN}$ are positive for anti-PLA2R antibodies, while those with tumor-associated $\mathrm{MN}$ are often negative for anti-PLA2R antibodies (28-30).

The final diagnosis of tumor-associated nephropathy is currently judged mainly by diagnostic treatment, and the symptoms of nephrotic syndrome can be significantly improved after tumor resection. What is special about the glomerular damage caused by renal cancer is that in the case of patients with renal insufficiency, surgical complications may lead to further deterioration of postoperative renal function. How should we choose? It is recommended that surgeons strictly control the indications and contraindications according to the specific conditions of different patients, and try to preserve nephron sparing surgery for patients with indications while reducing intraoperative errors and strengthening postoperative management to avoid serious complications and minimize the possibility of worsening renal function.

Our conclusion of this case, the diagnosis of tumorassociated glomerular diseases, is due to the following reasons: (I) the large size of the tumor, there should be a tumor's history for many years, while the lower extremity edema is only 2 months; (II) other factors which could cause glomerular damage are excluded; (III) the pathological, immunohistochemical and special staining results as well as other biochemical indicators of this case; (IV) after the primary tumor resection, the tumor load greatly reduced, the symptoms and examination results of nephrotic syndrome gradually improved. And we also call for the detection of tumor-associated antigens in the glomerular lesion when suspected tumor-associated glomerular disease, which is ignored in this case.

The strengths of this case report are that we chose to treat the tumor with partial nephrectomy first, protecting the kidney function to the greatest extent possible, and also avoiding the tumor enlargement caused by the treatment of nephrotic syndrome. But this is still limited for other patients who have a large tumor not suitable for partial nephrectomy.

According to the above, there is a strong pathogenic relationship between glomerular disease and malignant tumor. Paraneoplastic glomerulonephritis is a rare secondary cause of glomerulonephritis and a complication of cancer, which is a nephrologist and oncologists' challenge. Although any pathological type of GN may be associated with cancer, $\mathrm{MN}$ patients, especially those older than 60 years, have a more frequent risk of solid tumors. As is pointed out by some studies, the diagnosis of $\mathrm{MN}$ is often preceded by related cancers, sometimes even years (28). Therefore, timely identification of paraneoplastic glomerulonephritis and subsequent discovery of undiagnosed malignant tumors can attribute to the effective treatment and satisfied prognosis.

How the patients diagnosed with glomerular disease and whether they should be screened for cancer are still issues discussed by nephrologists. Currently, the Kidney Science Association has not recommended any guidelines. In the absence of internationally recognized guidelines and evidence-based indications, we believe that a focused examination will enable us to identify most of these cancers in patients, especially in elderly patients. It should include a complete family and patient history, with careful physical 
examinations. When there is no specific cancer suspicion, tumor screening is very vital in the general population for high-risk patients, such as fecal occult blood and colonoscopy for patients over 50 years old, mammography for women over 40 years old, and prostate-specific antigen testing for men over 50 years old. Patients with a history of smoking should be evaluated by chest X-ray or chest computed tomography to rule out lung cancer. For patients with MCD, especially those with systemic symptoms or resistance to traditional MCD treatment, it is highly necessary to check Hodgkin's lymphoma. If there is any suggestion of occult lymphoma or leukemia, bone marrow biopsy should be performed.

There is an urgent need to identify prognostic biomarkers for paraneoplastic glomerulonephritis in blood, urine or kidney biopsy samples by proteomics or other methods to facilitate early diagnosis of the disease.

A multidisciplinary approach involving nephrologists, oncologists, and other caregivers is necessary to treat primary malignant tumors and paraneoplastic glomerulonephritis. The main treatment is to treat the cause (i.e., the primary tumor lesions). The symptomatic treatment of nephrotic syndrome is the same as that of noncancer patients. After successful treatment, renal function tests, urine analysis and urine protein should be followed up for a long time. Any recurrence of glomerular lesions may indicate the cancer recurrence. Similarly, any malignant tumor recurrence should remind the doctor to look for a recurrence of paraneoplastic glomerulonephritis.

With the advent of the precision medicine era, genetic testing will play an important role in the diagnosis and treatment of diseases. Some drugs have been developed that target specific genes to improve the quality of life of patients. Further research is needed to determine the association between glomerular disease and cancer, as well as the underlying molecular mechanisms. This will make it possible to identify the underlying genomics of cancers associated with glomerular diseases and develop new therapeutic strategies, that are extremely important for some patients who are unable to perform surgery, filling the existing blank area.

\section{Acknowledgments}

Funding: None.

\section{Footnote}

Conflicts of Interest: All authors have completed the ICMJE uniform disclosure form (available at http://dx.doi. org/10.21037/tau.2020.03.41). The authors have no conflicts of interest to declare.

Etbical Statement: The authors are accountable for all aspects of the work in ensuring that questions related to the accuracy or integrity of any part of the work are appropriately investigated and resolved. The case study was approved by the Ethics Committee of The Second Affiliated Hospital of Chongqing Medical University, and the patient provided informed consent.

Open Access Statement: This is an Open Access article distributed in accordance with the Creative Commons Attribution-NonCommercial-NoDerivs 4.0 International License (CC BY-NC-ND 4.0), which permits the noncommercial replication and distribution of the article with the strict proviso that no changes or edits are made and the original work is properly cited (including links to both the formal publication through the relevant DOI and the license). See: https://creativecommons.org/licenses/by-nc-nd/4.0/.

\section{References}

1. Galloway J. Remarks on Hodgkin's disease. Br Med J 1922;2:1201-8.

2. Ronco PM. Paraneoplastic glomerulopathies: new insights into an old entity. Kidney Int 1999;56:355-77.

3. Lee JC, Yamauchi H, Hopper J Jr. The association of cancer and the nephrotic syndrome. Ann Intern Med 1966;64:41-51.

4. Bacchetta J, Juillard L, Cochat P, et al. Paraneoplastic glomerular diseases and malignancies. Crit Rev Oncol Hematol 2009;70:39-58.

5. Vaziri ND. Disorders of lipid metabolism in nephrotic syndrome: mechanisms and consequences. Kidney Int 2016;90:41-52.

6. Barata PC, Rini BI. Treatment of renal cell carcinoma: Current status and future directions. CA Cancer J Clin 2017;67:507-24.

7. Pani A, Porta C, Cosmai L, et al. Glomerular diseases and cancer: Evaluation of underlying malignancy. J Nephrol 
2016;29:143-52.

8. Leeaphorn N, Kue APP, Thamcharoen N, et al. Prevalence of cancer in membranous nephropathy: a systematic review and meta-analysis of observational studies. Am J Nephrol 2014;40:29-35.

9. Cudkowicz ME, Sayegh MH, Rennke HG. Membranous nephropathy in a patient with renal cell carcinoma. Am J Kidney Dis 1991;17:349-51.

10. Jiang WX, Wei LW, Yan WG, et al. A case report of paraneoplastic syndrome of renal cell carcinoma secondary to membranous nephropathy. Chin J Urol 2018,39:709-10.

11. Kuroda I, Ueno M, Okada H, et al. Nephrotic syndrome as a result of membranous nephropathy caused by renal cell carcinoma. Int J Urol 2004;11:235-8.

12. Kerpen HO, Bhat JG, Feiner HD, et al. Membranes nephropathy associated with renal cell carcinoma. Evidence against a role of renal tubular or tumor antibodies in pathogenesis Am J Med 1978;64:863-7.

13. Stein HD, Yudis M, Sirota RA, et al. Membranous nephropathy associated with renal cell carcinoma. Am J Kidney Dis 1993;22:352.

14. Fujita Y, Kashiwagi T, Takei H, et al. Membranous nephropathy complicated by renal cell carcinoma. Clin Exp Nephrol 2004;8:59-62.

15. Nunez S, Konstantinov KN, Servilla KS, et al. Association between scleroderma, renal cell carcinoma and membranous nephropathy. Clin Nephrol 2009;71:63-8.

16. Mimura I, Tojo A, Kinugasa S, et al. Renal cell carcinoma in association with IgA nephropathy in the elderly. Am J Med Sci 2009;338:431-2.

17. Accidental Discovery of Renal Cell Carcinoma with Renal Biopsy in a Patient Presenting with Nephrotic Syndrome: When Luck Saves Lives. Available online: https://www. ajkd.org/article/S0272-6386(18)30415-3/fulltext

18. Woodrow G, Innes A, Ansell ID, et al. Renal cell carcinoma presenting as nephrotic syndrome. Nephron 1995;69:166-9.

19. Le Berre L, Hervé C, Buzelin F, et al. Renal macrophage activation and Th2 polarization precedes the development of nephrotic syndrome in Buffalo/Mna rats. Kidney Int 2005;68:2079-90.

20. Takeda S, Chinda J, Murakami T, et al. Development of features of glomerulopathy in tumor-bearing rats: A potential model for paraneoplastic glomerulopathy. Nephrol Dial Transplant 2012;27:1786-92.

21. Wakashin M, Wakashin Y, Iesato K, et al. Association of gastric cancer and nephrotic syndrome. An immunologic study in three patients. Gastroenterology 1980;78:749-56.

22. Haskell LP, Fusco MJ, Wadler S, et al. Crescentic Glomerulonephritis Associated With Prostatic Carcinoma: Evidence of Immune-Mediated Glomerular Injury. Am J Med 1990;88:189-92.

23. Olson JL, Philips TM, Lewis MG, et al. Malignant melanoma with renal dense deposits containing tumor antigens. Clin Nephrol 1979;12:74-82 .

24. Couser WG, Wagonfeld JB, Spargo BH, et al. Glomerular deposition of tumor antigen in membranous nephropathy associated with colonic carcinoma. Am J Med 1974;57:962-70.

25. Hoxha E, Wiech T, Stahl PR, et al. A Mechanism for Cancer-Associated Membranous Nephropathy. N Engl J Med 2016;374:1995-6.

26. Mustonen J. IgA glomerulonephritis and associated diseases. Ann Clin Res 1984;16:161-6.

27. Taniguchi K, Fujioka H, Torashima Y, et al. Rectal cancer with paraneoplastic nephropathy: association of vascular endothelial growth factor. Dig Surg 2004;21:455-7.

28. Lefaucheur C, Stengel B, Nochy D, et al. Membranous nephropathy and cancer: epidemiologic evidence and determinants of high-risk cancer association. Kidney Int 2006;70:1510-7.

29. Ponticelli C, Glassock RJ. Glomerular diseases: membranous nephropathy-a modern view. Clin J Am Soc Nephrol 2014;9:609-16.

30. Qin W, Beck LH Jr, Zeng C, et al. Anti-phospholipase A2 receptor antibody in membranous nephropathy. J Am Soc Nephrol 2011;22:1137-43.
Cite this article as: Song F, Liu C, Zhang J, Hu Z. Renal cell carcinoma with nephrotic syndrome: a case report and literature review. Transl Androl Urol 2020;9(3):1459-1465. doi:10.21037/ tau.2020.03.41 